\title{
TRASCOLACIÓN Y PÉRDIDAS POR INTERCEPTACIÓN EN UN BOSQUE DE ROBLE MELOJO DEL SISTEMA CENTRAL
}

\author{
C. MORÁN TEJEDA, J. MARTÍNEZ FERNÁNDEZ, \\ V. HERNÁNDEZ SANTANA y A. CANO CRESPO
}

Departamento de Geografía. Universidad de Salamanca Cervantes, 3. 37002 Salamanca

Correo electrónico de contacto: cmoran@usal.es

RESUMEN. La interceptación, como proceso responsable del volumen de precipitación que no llega a la superficie del suelo de las formaciones vegetales, ha sido objeto de numerosos estudios en diferentes tipos de vegetación. Sin embargo, en la literatura científica se pueden encontrar ciertas lagunas en los estudios de interceptación bajo diferentes coberturas vegetales. Es el caso del roble melojo (Quercus pyrenaica Willd.) al que, aún siendo una de las especies forestales que más territorio ocupa en la Península Ibérica, se le han dedicado escasos trabajos sobre interceptación.

En este sentido se ha abordado el estudio de los volúmenes de trascolación en una pequeña cuenca de media montaña tapizada por un bosque de roble melojo en el Sistema Central. Para ello se dispusieron un total de 64 colectores de trascolación en 4 parcelas experimentales que atendian a diferentes estados de madurez del bosque. En el análisis se utilizaron los datos de 25 meses de estudio, con lo que se pudieron muestrear dos periodos estacionales completos. Se analizaron por separado los periodos con y sin biomasa foliar.

El porcentaje medio de trascolación recogida en la zona de estudio con respecto a la precipitación incidente supone que al menos el 14,9\% del agua caída en la cuenca se evapora nuevamente a la atmósfera y no pasa a formar parte del ciclo hidrológico superficial. En el periodo en el que el bosque tenía biomasa foliar, el porcentaje interceptado fue del $18 \%$ y en el periodo sin hojas del 11,9\%.

ABSTRACT. Interception, as the responsible process of the amount of precipitation never reaching the ground in vegetable communities, has been the object of several studies in various vegetation types, even though there are absences in the interception studies under some forested areas. This is the case of the Pyrenean oak (Quercus pyrenaica Willd.), one of the species that occupies more extension in the Iberian Peninsula, for which only a few studies have been carried out. 
In this sense, a study of the amount of throughfall in a small catchment covered by oak, has been conducted. To achieve this purpose, 64 throughfall collectors were placed in four different plots, which represent four different growth stages of the forest. In the analysis, the data monitored throughtout 25 months was used. This way, two complete seasonal periods could be sampled.

The average percentage of throughfall in the catchment yields 14,9\% of the total rainfall. This means that is evaporated to the atmosphere and is not part of the superficial hydrological cycle. In the period when the oaks have leaves, the average intercepted percentage was $18 \%$ and in the non-leaf period this percentage was the $11,9 \%$.

Palabras clave: interceptación de la lluvia, trascolación, Quercus pyrenaica, Sistema Central.

Key words: rainfall interception, throughfall, Quercus pyrenaica, Sistema Central.

Enviado el 24 de septiembre de 2007 Aceptado el 9 de mayo de 2008

\section{Introducción}

Desde la antigüedad se ha intentado entender el impacto que tienen los bosques en la hidrología y en la meteorología (Andréassian, 2004). Este interés se ha traducido en el estudio científico de las interacciones bosque-agua. La vegetación constituye uno de los componentes biológicos del ciclo hidrológico e influye en éste de diferentes maneras. Una de ellas es la interceptación, que es el agua que es retenida por la cubierta vegetal y que no llega al suelo, evaporándose nuevamente a la atmósfera y no llegando a formar parte del ciclo hidrológico de la superficie (Singh, 1992). Es el proceso por el cual la precipitación es detenida y retenida por la vegetación y se pierde por evaporación antes de alcanzar el suelo (UNESCO, World Meteorological Organization, 1992). Dependiendo de la naturaleza y densidad de la cubierta, una proporción de la lluvia puede ser interceptada por las hojas y ramas de la cubierta vegetal y temporalmente acumulada en su superficie (Ward y Robinson, 2000). La interceptación se calcula como la diferencia entre la precipitación total registrada fuera de la cubierta y la suma de la trascolación y de la escorrentía cortical, de manera que:

$$
\mathrm{I}=\mathrm{P}-(\mathrm{Tr}+\mathrm{Ec})
$$

Donde: I es la Interceptación en mm; P la precipitación total en mm; Tr la trascolación en $\mathrm{mm}$; y Ec la escorrentía cortical en mm.

La trascolación, o precipitación penetrante, es la parte de la precipitación que alcanza el suelo directamente a través de la vegetación, a través de los espacios entre las plantas y por el goteo de las hojas, ramas y tallos (UNESCO, WMO, 1992). La escorrentía cortical procede del agua retenida por la superficie de los troncos (Singh, 1992), que ha 
caído directamente sobre ellos, o proviene del flujo previamente incidente sobre las copas y que luego resbala por las ramas y llega a los troncos hasta entrar en el suelo por la base de los mismos.

La cantidad de precipitación que llega a la superficie del suelo depende de la cubierta vegetal. Esta cubierta intercepta parte de la precipitación y puede ser almacenada temporalmente en las hojas, ramas y tronco, desde donde puede evaporarse (Ward y Robinson, 2000). A este fenómeno se le conoce como pérdidas por interceptación.

Cuando se realiza un estudio de interceptación, hay que tener en cuenta una serie de factores que influyen en el fenómeno. Por un lado están los factores meteorológicos (Toba y Ohta, 2005), y dentro de estos aquellos que afectan en mayor medida al proceso de la interceptación son la lluvia (Crockford y Richardson, 2000; Singh, 1992), el viento (Neff, 1977; Gash y Stewart, 1977; Belmonte y Romero, 1999), la temperatura (Heras, 1972; Martínez y Navarro, 1996) y la radiación (Stewart, 1977). Por otro lado, el otro conjunto de factores más importantes que afectan a la interceptación son las características de la vegetación: tamaño de copa, forma, superficie y orientación de las hojas, ángulo de las ramas, tipo de corteza, densidad de la cubierta y fenología de las plantas (Cape et al., 1991; Tobón et al., 2000).

La interceptación es un proceso con una consideración creciente en los estudios de hidrología, debido a su importancia como proceso hidrológico de los sistemas forestales (Vrugt et al., 2003). Horton, en 1919, describió el fenómeno de la interceptación de las precipitaciones por la vegetación (Helvey y Patric, 1965) y desde entonces han sido muchos los trabajos de interceptación que se han realizado (Mateos, 2003).

Durante las últimas décadas, en la Península Ibérica se ha producido una expansión de los bosques debido a factores como el éxodo rural, el abandono de la agricultura y de las labores tradicionales de explotación. Según datos del Inventario Forestal Nacional, IFN (Ministerio de Agricultura, 1968-1974; Ministerio de Agricultura, Pesca y Alimentación, 1990-1997), la superficie arbolada de España aumentó un 18\% entre 1974 (primer IFN) y 1997 (segundo IFN). Los datos del tercer IFN, todavía en realización, indican que ese crecimiento persiste hasta la actualidad. Esta expansión y desarrollo tiene que suponer necesariamente una variación en el balance de agua, ya que el bosque es consumidor de recursos hídricos, en mayor medida que una zona de pastos o de labor agrícola, debido, por un lado a que intercepta más precipitación, y por otro, a las características y necesidades fisiológicas de los árboles (Calder, 1992).

En la Península Ibérica, han sido pocos los estudios publicados sobre interceptación en bosques de Quercus pyrenaica, a pesar de ser una especie muy abundante. A finales de la década de los 90 del siglo pasado, ocupaba más de 300000 ha como especie dominante y estaba presente en otras 70000 ha como acompañante (MAPA 1990-1997). Ibarra y Echeverría (2004) hicieron un estudio sobre la interceptación en la Sierra del Moncayo y compararon las pérdidas por interceptación en tres bosques de tres especies diferentes, una de las cuales era el roble melojo. Moreno et al. (1996), llevaron a cabo una investigación del ciclo de nutrientes en un bosque de $Q$. pyrenaica en el que hicie- 
ron un estudio del volumen de agua interceptada por la cubierta arbórea. Hay autores que han realizado trabajos sobre interceptación en otras quercinias en el territorio español. Calabuig (1978) fue el primero en estudiar el efecto que producía la encina (Quercus rotundifolia Lam.) en la redistribución de la precipitación. Bellot y Escarré (1991) estudiaron la interceptación por parte de la encina (Quercus ilex L.), pero basaron su estudio en los nutrientes aportados por la trascolación y la escorrentía cortical. Mateos (2003), también estudió la interceptación de las precipitaciones por parte de la encina ( $Q$. rotundifolia) en una dehesa, así como su variabilidad espacial. Fuera de la Península Ibérica se han llevado a cabo estudios de interceptación en especies de quercinias. Cape et al. (1991), entre las especies que utilizaron para llevar a cabo su comparación de la interceptación en varios bosques del Reino Unido, eligieron un bosque de roble albar (Quercus petraea Matt Liebl.). Carlyle-Moses y Price (1999) aplicaron un modelo sobre la interceptación en un bosque en el que dominaba el roble rojo (Quercus rubra L). Xiao et al. (2000) estudiaron la interceptación invernal por parte de dos bosques, uno de los cuales estaba formado por alcornoque (Quercus suber L.).

La escasez de información sobre una de las especies más importantes y más abundantes de la Península Ibérica hacen necesario el estudio detallado de la interceptación bajo la cobertura del $Q$. pyrenaica.

El objetivo general que se pretende alcanzar en el presente estudio es el de conocer el comportamiento de la precipitación cuando atraviesa el dosel de un bosque de roble melojo (Quercus pyrenaica) que se encuentra situado en la cara norte de la Sierra de Tamames (Salamanca), en el Sistema Central. En este sentido, se pretende estudiar cómo llega el agua de la lluvia al suelo después de traspasar la vegetación y en qué medida se ve afectado el volumen de precipitación por la presencia o no de biomasa foliar.

\section{2. Área de estudio}

El estudio se ha llevado a cabo en la Cuenca Experimental (C.E.) de Rinconada, localizada en la vertiente norte de la Sierra de Tamames, Sistema Central (provincia de Salamanca) (Fig. 1). La cuenca ocupa una extensión de 62 ha, su altitud oscila entre los 1140 y 1450 m y presenta una orientación suroeste-noroeste. Las condiciones climáticas de la zona son de tipo mediterráneo subhúmedo, con una temperatura media de $10^{\circ} \mathrm{C} \mathrm{y}$ una precipitación media anual alrededor de 1000 mm (Martínez-Fernández et al., 2005).

La cuenca está tapizada en un $68 \%$ por un melojar ( $Q$. pyrenaica) que se corresponde con la serie meso-supramediterránea, subhúmeda-húmeda, silicícola del roble melojo (Genisto falcatae-Querceto pyrenaicae) (Navarro y Valle, 1987). Este robledal ha sufrido una acusada presión antrópica durante gran parte del siglo XX, no sólo silvopastoril (talas, leñeo y pastoreo), sino también para el abastecimiento de los hornos de cal y el carboneo. Este proceso culminó en una tala generalizada a finales de la década de los 60 del siglo pasado. En los últimos 30 años la utilización del monte se ha limita- 


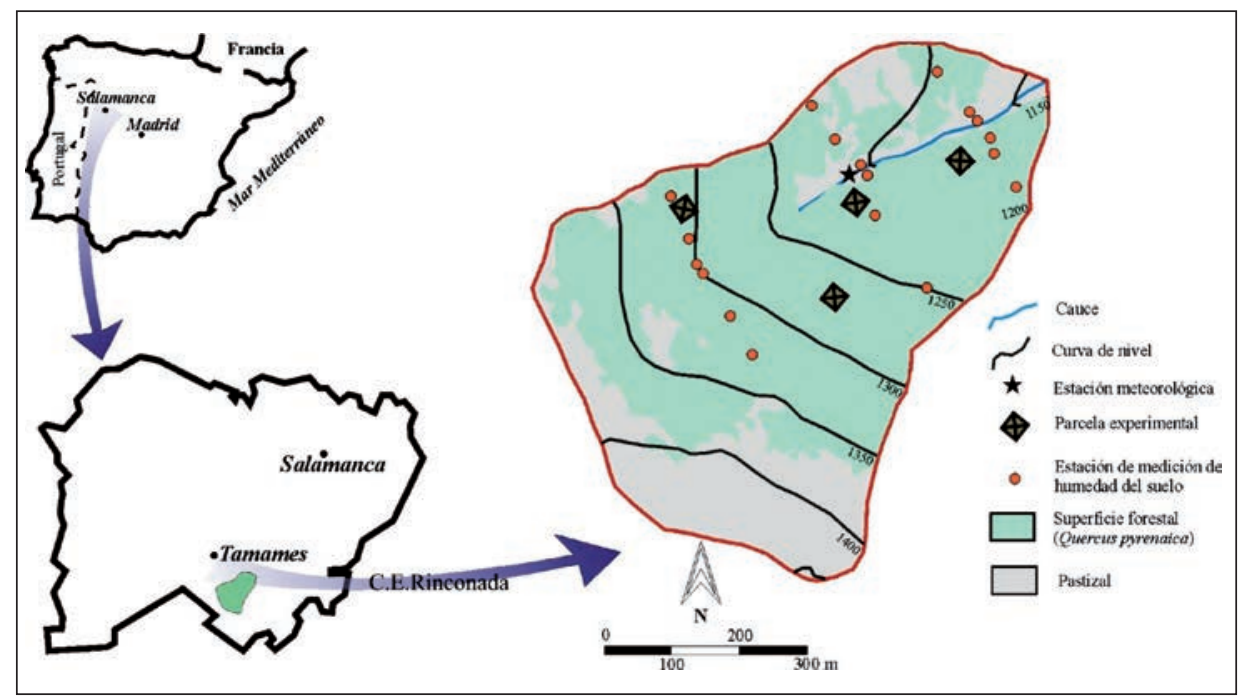

Figura 1. Localización de la Cuenca Experimental de Rinconada

do a un pastoreo extensivo de baja intensidad que ha permitido una recuperación significativa de la cubierta arbórea. Actualmente el bosque es homogéneo, con una alta densidad de pies por hectárea (2300 aproximadamente).

\section{Materiales y métodos}

La C.E. de Rinconada cuenta con una estación meteorológica (EMMA), instalada en un claro del bosque con la distancia necesaria a los árboles para que éstos no interfieran en las medidas. Esta estación meteorológica registra datos de las principales variables climáticas, de manera automática con un intervalo temporal de 10 minutos.

La precipitación fue recogida en la EMMA por dos dispositivos. Por un lado, con un pluviómetro automático (ARG 100 Campbell Scientific $\left.{ }^{\circledR}\right)$ y por otro, con un pluviómetro totalizador.

El 10 de julio de 2002 se instalaron cuatro dispositivos para realizar un primer test de la trascolación y la escorrentía cortical. Los colectores para medir la trascolación son totalizadores que consisten en un tubo de plástico de $130 \mathrm{~cm}$ colocado verticalmente, cuya boca lleva instalado un embudo de $8.5 \mathrm{~cm}$ de diámetro que canaliza el agua a través de un tubo hasta un colector de 2 litros de capacidad situado en una caja colocada bajo tierra para minimizar la evaporación.

Los dispositivos de escorrentía cortical instalados consisten en un tubo de plástico enrollado en espiral alrededor del tronco de un árbol. El tubo está cortado de manera lon- 
gitudinal hasta aproximadamente la mitad de su longitud para recoger así el agua que escurre por el tronco. Al final de este tubo, se conectó otro para canalizar el agua hacia un recipiente de 2 litros de capacidad que está metido en una caja y ésta a su vez enterrada en el suelo.

Tanto los colectores de escorrentía cortical, como los pluviómetros de trascolación, se dispusieron en cuatro parcelas previamente seleccionadas. Cada una de las parcelas, de $400 \mathrm{~m}^{2}$, se había elegido con un criterio dasométrico en una campaña realizada en abril de 2002 (Tabla 1). Cada parcela, atendía a un estado diferente de desarrollo del bosque. De esta forma, quedó establecida la clasificación de la siguiente manera: R1: robledal viejo, R2: robledal maduro aclarado reciente, R3: robledal maduro aclarado antiguo, R4: robledal joven sin aclarar

Tabla 1. Características de las parcelas experimentales

\begin{tabular}{|c|c|c|c|c|}
\hline & R1 & $\mathbf{R 2}$ & $\mathbf{R 3}$ & $\mathbf{R 4}$ \\
\hline Área de la parcela $\left(\mathrm{m}^{2}\right)$ & 109,18 & 124,63 & 98,94 & 111,18 \\
\hline Altitud (m) & 1310 & 1286 & 1244 & 1216 \\
\hline Pendiente $\left({ }^{\circ}\right)$ & 10 & 15 & 14 & 12 \\
\hline Orientación & $\mathrm{E}$ & NNE & $\mathrm{N}$ & NNE \\
\hline Densidad (pies ha-1) & 1975 & 1050 & 1950 & 4475 \\
\hline DBH medio $(\mathrm{cm})^{\mathrm{a}}$ & 11,70 & 13,70 & 12,60 & 8,10 \\
\hline Altura media árboles $(\mathrm{m})$ & 7,40 & 10,77 & 9,46 & 6,21 \\
\hline $\operatorname{LAI}\left(\mathrm{m}^{2} \mathrm{~m}^{-2}\right)^{\mathrm{b}}$ & 3,40 & 1,65 & 4,16 & 3,38 \\
\hline
\end{tabular}

${ }^{a}$ DBH, Diameter Breast Height

${ }^{b}$ LAI, Leaf Area Index

Después de aproximadamente 19 meses de seguimiento de los datos obtenidos mediante estos dispositivos, se abordó el estudio de la interceptación de una manera más detallada, poniendo más énfasis en el estudio de la trascolación, ya que se observó que la escorrentía cortical suponía un volumen muy pequeño de agua. Como media de las cuatro parcelas, el resultado fue que el flujo cortical suponía un porcentaje muy bajo $(0,78 \%)$ del volumen total precipitado en la cuenca (R1, 0,79\%; R2, 1,03\%; R3, 0,44\%; R4, 0,86\%). A pesar de que sólo se había escogido un árbol por parcela (representativo de la clase diametral media), y de que con más individuos muestreados es posible que los porcentajes hubieran variado, es probable que esta variación no fuera tan reseñable y en ningún caso la escorrentía cortical supondría un porcentaje significativo de la precipitación.

En cada parcela se seleccionó una subparcela de aproximadamente $100 \mathrm{~m}^{2}$. Es importante señalar que todas las parcelas tienen prácticamente la misma orientación y 
que, por tanto, se pueden descartar diferencias pluviométricas que puedan estar relacionadas con este factor. Se dispusieron en una malla regular 16 pluviómetros de trascolación, con una distancia de 2.6 m entre sí y con origen a un metro del límite superior y de uno de los laterales de las parcelas. Esta disposición se considera apropiada, ya que el bosque es bastante homogéneo y el tamaño de los árboles no es muy grande si se compara con el tamaño de la parcela (Lloyd y Marques, 1988).

Los colectores de trascolación están formados por recipientes de polietileno de $29 \mathrm{~cm}$ de altura, con un embudo en la boca de $8,5 \mathrm{~cm}$ de diámetro, lo que hace que la superficie receptora sea de $56,75 \mathrm{~cm}^{2}$. La altura del embudo que recoge el agua está entre 34 y $50 \mathrm{~cm}$ sobre el suelo. El hecho de que se hayan colocado a esta altura, minimiza los efectos del viento, pues con la altura, el viento puede distorsionar las mediciones (Sevruk, 1996). Además, así se puede también computar la interceptación de los estratos bajos de los robles. Por otro lado están a una altura suficiente como para que las gotas que impactan contra el suelo no salpiquen y caigan en la boca del pluviómetro (Iroumé y Huber, 2002).

De acuerdo con Tobón et al. (2000), para minimizar la evaporación de los colectores, se colocó un tubo de plástico que va desde la boca del embudo hasta el fondo del colector.

El 12 de marzo de 2004 se instaló en cada parcela un pluviómetro automático en un punto elegido de manera aleatoria para tener un registro detallado de la cantidad y de la intensidad con la que el agua traspasa el dosel arbóreo.

\section{Resultados y discusión}

\subsection{Volúmenes de trascolación en la C.E. de Rinconada}

Para determinar el volumen de trascolación medio de la cuenca se han utilizado los datos de los episodios de precipitación ocurridos entre el 1 de abril de 2004 y el 31 de abril de 2006. Hay que destacar que durante los años en los que los dispositivos han estado funcionando, se ha dado un comportamiento pluviométrico especial, ya que la precipitación en la cuenca ha estado muy por debajo de la media. La precipitación media registrada en la estación meteorológica automática en 2004 fue de 656,8 mm, en 2005 de 660,8 mm y desde enero hasta abril de 2006, ambos incluidos, de 320,3 mm. Si se comparan estos datos con la precipitación media de la serie obtenida en el observatorio meteorológico del Instituto Nacional de Meteorología situado en el pueblo de Rinconada (939 mm), con 32 años de observaciones, la precipitación en 2004 supuso un 30,1\% menos que la media y en 2005 un $29,6 \%$ menos que la media. Esto supuso, simplemente, que se registraran menos episodios de precipitación que si se hubiesen dado condiciones normales y cabe pensar que esta circunstancia no interfiere en los resultados obtenidos.

Después de 25 meses de periodo de observaciones y muestreo del volumen de trascolación en la Cuenca Experimental de Rinconada, se ha obtenido un valor medio de tras- 
colación para las cuatro parcelas de la cuenca del 14,9\% El coeficiente de variación (CV) medio de los 25 meses de estudio en las cuatro parcelas experimentales fue del $0,47 \%$. En la Fig. 2 se puede ver el diferente porcentaje de agua que no ha sido trascolado en cada parcela, referido a las medias mensuales. Así como la precipitación mensual registrada en la estación meteorológica durante todo el periodo de estudio. Se puede decir que, normalmente, los mayores porcentajes de interceptación se alcanzan en los meses de verano. Este hecho es comprensible, ya que es el periodo en el que además de existir biomasa foliar, se dan los valores más altos de radiación y por tanto de evaporación.

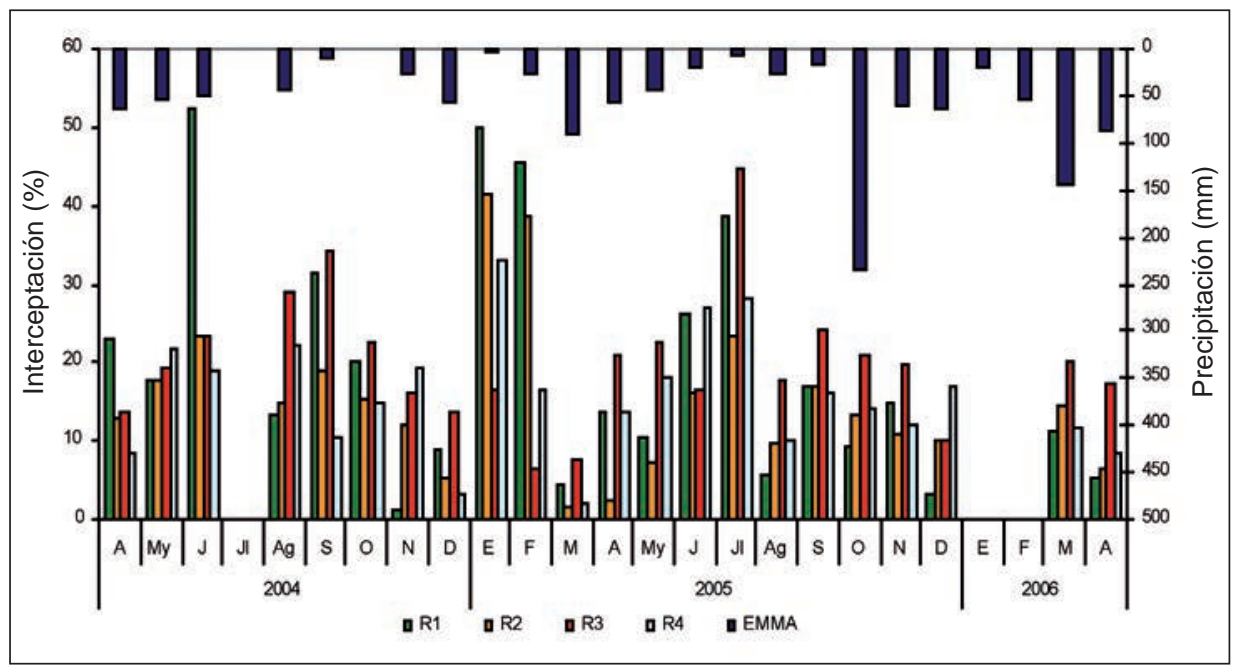

Figura 2. Porcentaje de interceptación en las diferentes parcelas de investigación durante todo el periodo estudiado

El mes en el que más trascolación media se obtuvo en las cuatro parcelas fue enero de 2005 , con un $35,4 \%$ y un CV del 18,9\%. El porcentaje mensual más alto de precipitación que no llego al suelo se produjo en la parcela R1 en el mes de junio de 2004, con un valor del 52,3\% (CV 21,6\%). Este dato de interceptación, a pesar de parecer excesivamente elevado, es comprensible si se tiene en cuenta que la precipitación que se produjo en la cuenca de Rinconada en ese mes fue aportada por dos episodios tormentosos, uno el 6 y otro el 9 de junio. Por tanto, es posible que en la parcela R1 precipitase menos que en la estación meteorológica, debido a la alta variabilidad espacial que presentan este tipo de fenómenos tormentosos (Zimmermann et al., 2001). El valor más bajo de flujo no trascolado se produjo, en la parcela R1 en el mes de noviembre de 2004, con un valor de $1,5 \%$ (CV 38,6\%). Un valor de interceptación bajo debido a que 5 colectores de trascolación recogieron más flujo de agua que el flujo de precipitación registrado en la estación meteorológica, lo que hace pensar que se dieron fenómenos de coalescencia. 
Se analizó por separado el periodo en el que el bosque se encontraba con biomasa foliar y el periodo en el que estaba carente de hojas. Los datos utilizados para determinar la trascolación en el periodo de actividad vegetativa corresponden a los meses de mayo a octubre, ambos inclusive. Para el periodo de dormición se usaron los datos de noviembre a abril. De esta manera, se pudo comprobar de qué manera afectaba en el volumen de agua recibida por el suelo del bosque la presencia o no de hojas en los árboles. Así, se obtuvieron los porcentajes de trascolación en cada parcela y en cada periodo con respecto a la estación meteorológica (Tablas 2 y 3).

Tabla 2. Interceptación en la Cuenca Experimental de Rinconada en el periodo de ausencia de hojas en los árboles

\begin{tabular}{|c|c|c|c|c|}
\hline \multicolumn{5}{|c|}{ Periodo Sin Hojas } \\
\hline \multirow[t]{2}{*}{2004} & \multicolumn{4}{|c|}{ \% Interceptación } \\
\hline & R1 & $\mathbf{R} 2$ & $\mathbf{R 3}$ & R4 \\
\hline $\mathrm{Ab}$ & 23,1 & 12,9 & 15,3 & 8,9 \\
\hline $\mathrm{N}$ & 1,5 & 12,2 & 20,6 & 19,4 \\
\hline $\mathrm{D}$ & 9,0 & 5,5 & 13,8 & 3,5 \\
\hline \multicolumn{5}{|l|}{2005} \\
\hline $\mathrm{E}$ & 46,7 & 41,7 & 33,3 & 33,3 \\
\hline $\mathrm{F}$ & 45,5 & 38,8 & 28,4 & 16,8 \\
\hline M & 4,9 & 1,9 & 8,0 & 2,4 \\
\hline $\mathrm{Ab}$ & 13,9 & 2,8 & 21 & 14,1 \\
\hline $\mathrm{N}$ & 15,2 & 11,2 & 19,2 & 12,2 \\
\hline $\mathrm{D}$ & 3,6 & 10,3 & 22,1 & 17,1 \\
\hline \multicolumn{5}{|l|}{2006} \\
\hline \multicolumn{5}{|l|}{$\mathrm{E}^{*}$} \\
\hline $\mathrm{F}^{*}$ & & & & \\
\hline M & 11,5 & 14,9 & 21,3 & 11,9 \\
\hline $\mathrm{Ab}$ & 5,6 & 6,6 & 17,4 & 8,6 \\
\hline
\end{tabular}

* Meses en los que no se ha producido precipitación y por lo tanto no ha habido interceptación. 
Tabla 3: Interceptación en la Cuenca Experimental de Rinconada

en el periodo de biomasa foliar

\begin{tabular}{|l|r|r|r|c|}
\hline \multicolumn{5}{|c|}{ Periodo Con Hojas } \\
\hline $\mathbf{2 0 0 4}$ & \multicolumn{4}{|c|}{ \% Interceptación } \\
\hline & R1 & R2 & R3 & R4 \\
\hline My & 17,9 & 18,0 & 19,6 & 21,9 \\
J & 52,3 & 23,7 & 23,7 & 19,3 \\
J1* & & & & \\
Ag & 13,5 & 15,3 & 29,2 & 22,2 \\
S & 31,5 & 19,0 & 34,5 & 10,7 \\
O & 20,2 & 15,6 & 22,9 & 15,2 \\
\hline 2005 & & & & \\
\hline My & 10,6 & 7,7 & 22,6 & 18,3 \\
J & 26,5 & 16,3 & 16,8 & 27,2 \\
J1 & 38,8 & 23,8 & 45,0 & 28,3 \\
Ag & 6,1 & 9,8 & 18,1 & 10,4 \\
S & 17,1 & 17,2 & 24,2 & 16,2 \\
O & 9,4 & 13,6 & 21,2 & 14,1 \\
\hline
\end{tabular}

* Meses en los que no se ha producido precipitación y por lo tanto no ha habido interceptación.

En el periodo con hojas, de manera general y para las cuatro parcelas estudiadas, el volumen medio de trascolación recogido fue de $608,7 \mathrm{~mm}$. El promedio trascolado de las cuatro parcelas supuso el $82 \%$ de la precipitación caída en la estación meteorológica $(742,3 \mathrm{~mm})$. Esto supuso que, como promedio para las cuatro parcelas, el 18\% (CV $1,26 \%)$ no llegó al suelo en forma de trascolación. De las cuatro parcelas, el porcentaje más alto de flujo no trascolado se dio en R3 (22,5\% con un CV del 1,17\%) y el más bajo en R2 (15,2\% con un CV del 0,91\%). La parcela R3 (Tabla 1) es la que presenta el valor más alto de Leaf Area Index (LAI) de las cuatro $\left(4,16 \mathrm{~m}^{2} \mathrm{~m}^{-2}\right)$ y por tanto es la que tiene mayor cobertura. Por el contrario, la parcela R2 es la que más bajo tiene el LAI (1,65 $\mathrm{m}^{2} \mathrm{~m}^{-2}$ ) y por tanto la que tiene una menor cobertura. De esta manera, la mayor o menor cobertura puede ser el factor que determine la mayor o menor llegada de agua en forma de trascolación a la superficie del suelo del bosque. Excepto en la parcela R1, cuyo valor porcentual de agua que no llegó al suelo como trascolación se registró en junio de 2004 $(52,3 \%)$, el máximo porcentaje de flujo no trascolado en las parcelas se alcanzó en el mes de julio de 2005. Los altos porcentajes registrados en estos meses de verano se pueden explicar, además, de por la existencia de biomasa foliar y por la elevada tasa de eva- 
poración de los meses de verano, por la naturaleza de las precipitaciones, que se dieron en forma de fenómenos tormentosos. Por tanto la variabilidad espacial asociada a la cantidad de precipitación que cayó sobre la cuenca pudo ser elevada y es posible que se diese mayor cantidad de precipitación en unas zonas que en otras. De la misma manera se puede explicar, pero en el caso contrario, las mínimas tasas de interceptación registradas en la parcela R1 y R4 en el mes de agosto de 2005. En la parcela R2 el mínimo se dio en el mes de mayo de 2005 (7,7\% con un CV del 9,1\%), mientras que en la parcela R1 se obtuvo el tercer valor más bajo de la parcela para todo el periodo $(10,6 \%$ con un CV del 13,1\%). Es significativo que esto ocurriese en las dos parcelas que están situadas a mayor altitud y por tanto más alejadas de la estación meteorológica y no en las dos parcelas más próximas a ésta (en la parcela R3 hubo un 22,6\% con un CV del 9,1\% y en la parcela R4 un 18,3\% y un CV del 12,8\%). Se puede pensar que existió un gradiente de precipitación y que cayó más agua en la parte baja de la cuenca que en la alta. Otra posibilidad es la de suponer que existieron puntos de coalescencia sobre los colectores e hicieron que la media de la parcela se viese aumentada y por tanto el porcentaje de flujo trascolado fuese mayor. Pudo deberse a esta hipótesis, ya que en la parcela R1 tres colectores de trascolación tuvieron más cantidad de agua que la precipitación recogida en la estación meteorológica. En la parcela R2, también tres colectores presentaron más precipitación que la registrada en la estación meteorológica. En las parcelas R3 y R4 sin embargo no se produjo coalescencia sobre ninguno de los pluviómetros de las parcelas.

Durante el periodo en el que el bosque estuvo sin biomasa foliar, el volumen de trascolación medio de las cuatro parcelas fue de 569,9 mm (excluyendo el agua recogida de los colectores por la aportación de la fusión de la nieve en los meses en los que ocurrió este tipo de precipitación). El promedio del porcentaje de flujo trascolado en las parcelas fue del $88,1 \%$, lo que supone que como media en la cuenca, el 11,9\% (CV 1,0\%) no llegó al suelo en forma de trascolación. De las cuatro parcelas, el porcentaje medio más alto de flujo no trascolado se dio en R3 (15,4\% con un CV del 1,1\%) y el más bajo en R2 (10,2\% con un CV del 0,9\%). Es comprensible que durante la época del año en la que no hay hojas en los árboles, la cobertura no constituya un factor predominante para explicar la mayor o menor cantidad de agua que llega a la superficie del suelo en forma de trascolación. En las cuatro parcelas, el mayor porcentaje de flujo interceptado se dio en el mes de enero de 2005, debido a la escasa precipitación registrada durante ese mes. En las parcelas R3 y R4, los meses en los que menos porcentaje se registró fueron febrero y marzo de 2005, respectivamente, mientras que en R1 y R2 fue en noviembre de 2004 y abril de 2005 respectivamente. En todas las parcelas, durante los meses mencionados hubo pluviómetros que recogieron más cantidad de agua que la que se recogió en la estación meteorológica, por lo que el porcentaje tan bajo registrado de flujo interceptado puede ser atribuible, en realidad, a procesos de coalescencia sobre los pluviómetros que hicieron que la media del volumen de trascolación de las parcelas se viese incrementada.

El hecho de que se haya encontrado un CV medio tan bajo en la serie de datos de interceptación, hace suponer que el dato medio de interceptación obtenido tiene el suficiente valor estadístico como para que sea representativo. Además, a pesar de que hay meses en los que el CV medio de las parcelas ha resultado elevado, en general se ha 
encontrado un CV medio bajo, tanto en los meses del periodo sin hojas, como en los meses del periodo con hojas. En este sentido, en el periodo en el que el bosque tiene biomasa foliar, se puede pensar que la cubierta vegetal es bastante homogénea y no se dan grandes diferencias en la llegada de agua al suelo del bosque.

Los porcentajes medios de trascolación que se han obtenido después de los 25 meses de estudio $(85,1 \%)$ están más cercanos a los valores que obtuvieron Moreno et al. (1996), en la Sierra de Gata, con un $84 \%$, que los obtenidos por Ibarra y Etxeverría (2004), en la Sierra del Moncayo, con un 88,6\%. Ambos estudios hechos en un bosque cuya especie dominante es el roble melojo (Q. pyrenaica).

La trascolación es el componente de la interceptación que más importancia cuantitativa presenta. Se puede decir que la trascolación varía dependiendo del tipo de bosque en el que se estudie, pero en ninguno de los estudios revisados ha supuesto un porcentaje inferior al 50\% de la precipitación total registrada fuera del bosque. En algunos casos se han obtenido porcentajes por encima del $80 \%$. Se puede decir que los valores más comunes registrados de trascolación variaban entre el $70 \%$ y el $80 \%$ de la precipitación total (Xiao et al., 2000; Rodrigo y Ávila, 2001; Iroumé y Huber, 2002; Crockford y Richardson, 2000).

Los valores obtenidos en este trabajo están próximos a los encontrados por otros autores en bosques caducifolios de quercinias. Cape et al. (1991) en un bosque de Quercus petraea obtuvieron un 82,4\%. Carlyle-Moses et al. (2004), en un bosque de Quercus glaucoides Mart., obtuvieron un $85 \%$ trascolados. Carlyle-Moses y Price (1999), en un bosque de Quercus rubra, calcularon un porcentaje trascolado del $79,7 \% \pm 3,5 \%$.

Los resultados obtenidos a partir de los volúmenes de trascolación recogidos en las cuatro parcelas experimentales demuestran el efecto que ejerce la biomasa foliar para interceptar agua, ya que se ha registrado una diferencia media del $6 \%$ en las cuatro parcelas. Es decir, en el periodo en el que hay hojas en los árboles, como media, llega al suelo un $6 \%$ menos de agua en forma de trascolación, que en el periodo en el que los árboles no tienen hojas en las ramas. Al mismo tiempo se demuestra que las hojas no son el único tipo de superficie vegetal que intercepta agua, ya que las diferencias entre los periodos con y sin hojas no son tan grandes como cabría esperar. Es de suponer, por tanto, que las ramas y los troncos de los árboles también son susceptibles de interceptar agua de una forma eficiente.

Al comparar parcelas ubicadas a diferentes altitudes, se ha podido constatar que en los meses invernales los porcentajes recogidos pueden llevar consigo un error, asociado al diferente estado de la precipitación (sólido-líquido) que cae sobre las parcelas. También se ha podido comprobar que la variabilidad espacial de la precipitación (sobre todo de la convectiva) puede hacer que en unas parcelas precipite más que en otras (Latrón et al., 2006). Esto puede dar lugar a que, al comparar los valores recogidos en los pluviómetros de las parcelas con un sólo punto de referencia, bajo determinadas condiciones climáticas, se detecten anomalías. 


\section{Conclusiones}

El dosel arbóreo supone un notable obstáculo para la llegada de agua al suelo de un bosque. Esto implica que la disponibilidad de recursos hídricos, no sólo para los árboles, sino también para los usos de un determinado territorio, se puede modificar considerablemente cuando existe una cubierta arbórea. La interceptación, como proceso responsable de esta modificación, ha hecho que sea un elemento imprescindible en los estudios sobre el balance hídrico de cuencas forestales.

Se ha llegado a la conclusión de que el principal componente de la interceptación, en términos cuantitativos, es la trascolación, ya que la escorrentía cortical, representa un porcentaje mínimo del total de precipitación registrada. El porcentaje medio de trascolación recogida durante los meses de estudio ha resultado similar al de estudios realizados en bosques caducifolios de características semejantes al de la cuenca de Rinconada. No obstante, han habido ciertas diferencias, si se compara con los estudios realizados en bosques de la misma especie.

Los resultados obtenidos han demostrado la influencia que ejerce en el porcentaje de flujo trascolado la presencia o ausencia de hojas en los árboles. Además, se ha podido comprobar la influencia del LAI en cuanto a la interceptación que se da en cada parcela en el periodo con hojas.

Ciertamente el agua es un recurso renovable. Sin embargo, y aunque parezca una contradicción, no es inagotable. Es por tanto condición indispensable hacer una buena gestión del recurso hídrico desde un punto de vista global, como un componente más del ecosistema. Para ello es imprescindible conocer los elementos implicados en la generación del recurso y los posibles factores que pueden desequilibrar su comportamiento.

\section{Agradecimientos}

Los autores quieren expresar su agradecimiento al Ministerio de Medio Ambiente (Proyecto RESEL) y al Ministerio de Ciencia y Tecnología (Proyecto REN2003-00381), sin cuya financiación no habría sido posible la realización de este trabajo.

\section{Referencias bibliográficas}

ANDRÉASSIAN, V. (2004). Waters and forests: from historical controversy to scientific debate. Journal of Hydrology, 291: 1-27.

BELlot, J., y EsCARRÉ, A. (1991). Chemical characteristics and temporal variations of nutrients in throughfall and stemflow of three species in Mediterranean holm oak forest. Forest Ecology and Management, 41: 125-135. 
BELMONTE SERRATO, F. (1997). Interceptación en bosque y matorral mediterráneo semiárido: Balance hídrico y distribución espacial de la lluvia neta. Tesis doctoral. Universidad de Murcia. 375 pp.

Calabuig, E. L., Gago-Gamallo, M. A. y Gómez-Gutiérrez, J. M. (1978). Influencia de la encina (Quercus ilex rotundifolia Lam.) en la distribución del agua de lluvia. Anuario del Centro de Edafología y Biología Aplicada de Salamanca, 4: 143.

CALDER, I. R. (1992). Hydrological effects of land-use change. Handbook of Hydrology (D.R. Maidment, Ed.). McGraw-Hill, 1350 pp.

Cape, J.N., Brown, A.H.F., Robertson, S.M.C., Howson, G., y Paterson, I.S. (1991). Interspecies comparisons of throughfall and stemflow at three sites in northern Britain. Forest Ecology and Management, 46: 165-177.

CARlYle-Moses, D.E. (2004). Throughfall, stemflow, and canopy interception loss fluxes in semi-arid Sierra Madre Oriental matorral community. Jornal of Arid Environments, 58: 181-202.

Carlyle-Moses, D.E. y Price, A. G. (1999). An evaluation of the Gash interception model in a northern hardwood stand. Journal of Hydrology, 214: 103-110.

CROCKFORD, R.H. y RiCHARDSON, D.P. (2000). Partitioning of rainfall into throughfall, stemflow and interception: effect of forest type, ground cover and climate. Hydrological Processes, 14: 2903-2920.

GASH, J.H.C. y STEWART, J.B. (1977). The evaporation from Thetford forest during 1975. Journal of Hydrology, 35: 385-396.

HelveY, J.D. Y PATRIC, J.H. (1965). Canopy and litter interception of rainfall by hardwoods of eastern United States. Water Resources Research, 1 (2): 193-206.

Heras, R. (1972). Manual de Hidrología. El ciclo hidrológico. Centro de Estudios Hidrográficos: Dirección General de Obras Hidráulicas, 872 pp., Madrid.

IBARRA, P. y ECHEVARRÍA, M.T. (2004). Relaciones clima, suelo y vegetación en la vertiente noreste del Moncayo. En: Geografía Física de Aragón. Aspectos generales y temáticos (Peña, J.L., Longares, L.A. y Sánchez, M., Eds). Universidad de Zaragoza e Institución Fernando el Católico, pp. 199-211, Zaragoza.

Iroumè, A. y Huber, A. (2002). Comparison of interception losses in a broadleaved native forest and a Pseudotsuga menziesii (Douglas fir) plantation in the Andes Mountains of southern Chile. Hydrological Processes, 16: 2347-2361.

Latron, J., Llorens, P. y Gallart, F. (2006). Spatial variability of rainfall and its influence on runoff modelling in a small mediterranean research catchment (Vallcebre, eastern Pyrenees). En: Uncertainties in the "monitoring-conceptualisa- 
tion-modelling" sequence of cathcment research. Proceedings of the eleventh biennial conference of the euromediterranean network of experimental and reperesentative basins (ERB) (Pfister, L., Matgen, P., van den Bos, R. y Hoffman, L., Eds.), pp.103-104, Luxemburgo.

Lloyd, C.R. y MARQues, F. (1988). Spatial variability of throughfall and stemflow measurements in Amazonian rainforest. Agricultural and Forest Meteorology, 42: 63-73.

Martínez, A., y NaVARro, J. (1996). Hidrología forestal, el ciclo hidrológico. Secretariado de publicaciones Universidad de Valladolid, pp. 286, Valladolid.

Martínez-Fernández, J., Ceballos, A., Casado, S., Morán, C. y Hernández, V. (2005). Procesos hidrológicos en una cuenca forestal del Sistema Central: cuenca experimental de Rinconada. Cuadernos de Investigación Geográfica, 31: 7-26.

MATEOS RodRíGUEZ, A.B., (2003). Interceptación de la lluvia por la encina en espacios adehesados. Universidad de Extremadura, Servicio de Publicaciones, 152 pp., Cáceres.

Ministerio de Agricultura (1968-1974). Primer inventario forestal de España. Ministerio de Agricultura, Dirección General de Montes, Caza y Pesca Fluvial, Madrid.

Ministerio de Agricultura, PesCa y Alimentación (1990-1997). Segundo inventario forestal nacional: 1986-1995. Ministerio de Agricultura, Pesca y Alimentación, Instituto Nacional para la Conservación de la Naturaleza, Madrid.

MorÁn TeJEDA, C. (2006). Trascolación en un bosque de roble melojo (Quercus pyrenaica Willd.) en la sierra de Tamames (Salamanca). Tesis de Licenciatura. Universidad de Salamanca, pp. 115, Salamanca.

Moreno, G., Gallardo, J. F., Schneider, K. y Ingelmo, F (1996). Water and bioelement fluxes in four Quercus pyrenaica forests along a pluviometric gradient. Annales des Sciences Forestieres, 53: 625-639.

NaVArro, F. y Valle, C.J. (1987): Castilla y León. En: La vegetación de España (Peinado, M. y Rivas., S., Ed.). Servicio de Publicaciones, Universidad de Alcalá de Henares, pp. 544, Alcalá de Henares.

Rodrigo, A. y ÁvilA, A. (2001). Influence of sampling size in the estimation of mean throughfall in two Mediterranean holm oak forest. Journal of Hydrology, 243: 216227.

SEVRUK, B. (1996). Adjustement of tipping-bucket precipitation gauge measurements. Atmospheric Research, 42: 237-246.

SingH, V.P. (1992). Elementary Hydrology. Prentice Hall, pp. 973, New Jersey. 
STEWART, J.B., 1977. Evaporation from the wet canopy of a pine forest. Water Resources Research, 13 (6): 915-921.

TовA, T. y OHTA, T. (2005). An observational study of the factors that influence interception loss in boreal and temperate forests. Journal of Hydrology, 313 (3-4): 208220.

Tobón Marín, C., Bouten, W. y Sevink, J. (2000). Gross rainfall and its partitioning into throughfall, stemflow and evaporation of intercepted water in four forest ecosystems in western Amazonia. Journal of Hydrology, 237: 40-57.

Unesco, World Metorological Organization (1992). International Glossary of Hydrology. Secretariat of the World Meteological Organization, pp. 413, Geneva.

VRugt, J.A., DeKKer, S.C., Bouten, W. (2003). Identification of rainfall inerception model parameters from measurements of throughfall and forest canopy storage. Water Resources Research, 39 (9): 1251.

Ward, R.C. y Robinson, M. (2000). Principles of Hydrology. McGraw-Hill, pp. 450, London.

Xiao, Q., McPherson, E.G., Ustin, S.L., Grismer, M.E. y Simpson, J.R. (2000). Winter rainfall interception by two mature open-grown trees in Davis, California. Hydrological Processes, 14: 763-784.

Zimmermann, E., RicCARdi, G., Pieroni, H. y Arraigada, M. (2001). Curvas de abatimiento areal de tormentas. Región Pampa ondulada (Santa Fe, Argentina). Cuadernos del Curiham, Phi-Unesco, 7 (1): 24-37. 